\title{
SÍNDROME DE BURNOUT EM DOCENTES DA EDUCAÇÃO BÁSICA
}

\author{
BURNOUT SYNDROME IN BASIC EDUCATION TEACHERS
}

Erik Cunha de Oliveira ${ }^{1}$

Gislainne Thaice da Silva Santos ${ }^{2}$

\begin{abstract}
RESUMO: Este estudo tem como objetivo discutir as possíveis causas da síndrome de burnout em docentes da educação básica. Trata-se de um artigo que resultou de uma pesquisa bibliográfica com realce para produções científicas que se referem a síndrome de burnout em professores, oriundo do mapeamento de pesquisas publicadas na área da Educação e Saúde. Estudos científicos revelam que a síndrome de burnout em docentes é um dos principais exemplos de distúrbio relacionado diretamente ao trabalho.
\end{abstract}

Palavras-chave: Adoecimento. Professor. Síndrome de burnout.

ABSTRACT: This study aims to discuss the possible causes of burnout syndrome in basic education teachers. This is an article that resulted from bibliographical research with emphasis on scientific productions that refer to the burnout syndrome in teachers, arising from the mapping of research published in Education and Health. Scientific studies reveal that the burnout syndrome in teachers it is one of the leading examples of a directly workrelated disorder.

Keywords: Illness. Teacher. Burnout syndrome.

\section{INTRODUÇÃO}

A síndrome de burnout é caracterizada como uma reação à tensão emocional crônica, que tem acometido, principalmente, profissionais que desenvolvem seu trabalho atendendo pessoas de forma direta, constante e emocional (MASLACH, 200I). Nesse sentido, o professor da educação básica se constitui como uma das categorias profissionais

\footnotetext{
${ }^{\text {I }}$ Psicólogo formado pela Universidade Salvador (UNIFACS). Mestrando em Educação pelo Programa de Pósgraduação em Educação da Universidade Tiradentes (UNIT). Bolsista CAPES. Email: erik.hf.r2@hotmail.com https://orcid.org/oooo-ooo2-0273-26r4.

${ }^{2}$ Graduanda em Fisioterapia pela Universidade Salvador (UNIFACS Email: gislainnethaicer5@hotmail.com https://orcid.org/oooo-oooz-9367-6274.
} 
mais sujeitas a adoecer devido as sensações dos excessos de obrigações que são destinados aos mesmos.

O processo de adoecimento que tem acometido os professores da educação básica tem sido fonte de pesquisa na área da saúde ocupacional em virtude das transformações ocorridas no cenário da educação brasileira que determina novas formas de gestão e organização escolar. Rajak e Chandra (2017) ressaltam que, nos últimos tempos, o desafio do professor não se limita apenas a ministrar conferências em sala de aula, ou seja, o docente é envolvido com outras responsabilidades acadêmicas, como atividades de investigação, organização de workshops e seminários, bem como gestão de estágios e outros programas de desenvolvimento.

Nesse sentido, Liorent e Ruiz-Calzado (2016) destacam que, a síndrome de burnout entre profissionais da educação tem sido um dos principais focos dos esforços dos pesquisadores, e estudos têm claramente mostrado que a profissão docente é uma das mais afetadas pelo burnout. No entanto, são necessárias mais investigações para que possa entender as dimensões do burnout e as várias características pessoais associadas à síndrome entre os profissionais da educação.

Em consideração ao objeto de estudo, este artigo tem como objetivo discutir as possíveis causas da síndrome de burnout em docentes da educação básica. Trata-se de um artigo que resultou de uma pesquisa bibliográfica com realce para produções científicas que se referem a síndrome de burnout em professores, oriundo do mapeamento de pesquisas publicadas na área da Educação e Saúde. Estudos científicos revelam que a síndrome de burnout em docentes é um dos principais exemplos de distúrbio relacionado diretamente ao trabalho.

Desta forma, tendo como base a compreensão da síndrome de burnout a partir das referências bibliográficas estudadas, o artigo será dividido em dois momentos: iniciando com a compreensão do conceito de síndrome de burnout, buscando assim, compreender os determinantes sociais que agravam a saúde do sujeito. E em seguida, discutir sobre síndrome de burnout na docência, apresentando dados teóricos sobre causas do adoecer em professores da educação básica. 


\section{Afinal, o que é a síndrome de burnout?}

A síndrome de burnout é um tipo particular de mecanismo de enfrentamento e de autoproteção diante do estresse gerado entre profissional-cliente, assim como na relação profissional-organização (GIL-MONTE, 2008). É, ainda, a consequência da relação precária entre os funcionários e seus respectivos locais de trabalho (LEITER E MASLACH, 2014).

O trabalho é uma atividade que pode ocupar parcela de tempo de cada indivíduo e do seu convívio em sociedade e, nem sempre possibilita realização profissional, ao contrário, pode causar problemas que vão desde a insatisfação até exaustão. Nesse caso, a síndrome de burnout pode ser compreendido como o conjunto de sinais e sintomas relacionados às situações de estresse laboral crônico, composta de três pilares de sustentação: a exaustão emocional, a despersonalização e a falta de realização pessoal (MASLACH, 2006).

Para Maslach, a exaustão emocional é caracterizada pelos sentimentos de estar sobrecarregado e exaurido de seus recursos físicos e emocionais, levando ao esgotamento de energia para investir nas situações que se apresentam no trabalho. A despersonalização é

considerada uma resposta à exaustão emocional, constituindo-se como estratégia de enfrentamento do indivíduo diante do estresse crônico. E a falta ou baixa realização profissional caracteriza-se por uma tendência do trabalhador em se autoavaliar de forma negativa, sentindo-se insatisfeito com seu desenvolvimento profissional, experimentando um declínio no sentimento de competência e na sua capacidade de interagir com as pessoas (MASLACH, 200I).

A síndrome de burnout é um processo individual, de evolução duradoura, podendo perdurar por anos ou décadas, com surgimento paulatino, cumulativo e progressivo em severidade. Muitas vezes, não é percebido pelo indivíduo que, na maioria das vezes, recusase a acreditar que está sendo acometido pela síndrome. Para Codo e Vasques-Menezes (1999) burnout consiste na síndrome da desistência, pois o indivíduo nessa situação, deixa de investir em seu trabalho e nas relações afetivas que dele decorrem e, aparentemente, torna-se incapaz de se envolver emocionalmente com o mesmo.

No entanto, a síndrome de burnout pode ser considerada como uma resposta crônica aos estressores interpessoais advindos da situação laboral, uma vez que o ambiente 
de trabalho e sua organização podem ser responsáveis pelo sofrimento e desgaste que acometem os profissionais, especificamente, os professores. É uma forma de adaptação que pode resultar em efeitos negativos tanto para a própria pessoa quanto para seu local de trabalho.

\section{SÍNDROME DE BURNOUT NA DOCÊNCIA}

Os profissionais da educação, especificamente aqueles que atuam na educação básica, têm sido alvo de diversas investigações em virtude das condições de trabalho. No Brasil, a categoria docente, segundo análise da produção científica realizada por Carlotto e Câmara (2008), é uma das que contempla um maior número de investigações. Tal fato pode ser explicado por uma submissão contínua desses profissionais a eventos estressantes como, a intensificação do trabalho, falta de reconhecimento na carreira, envolvimento emocional, acúmulo de funções, excesso de burocracias, entre outras.

Segundo a Organização Internacional do Trabalho (OIT), a profissão docente é considerada como uma das mais estressantes, com forte incidência de elementos que conduzem à síndrome de burnout (GIL-MONTE, 2008). Gil-Monte descreve em sua obra

sobre "A magnitude da relação entre burnout e o absenteísmo" que, o estresse está presente na vida dos docentes devido à rotina diária de trabalho e aos compromissos, podendo se manifestar de várias formas, por exemplo, alterações comportamentais. Esse fenômeno, que atinge professores de diferentes países, parece portar um caráter epidêmico mundial que extrapola as fronteiras nacionais.

Eventos estressantes está presente no quadro de problemas de saúde de várias profissões, inclusive em professores da educação básica. Os estressores podem representar uma adaptação inadequada à mudança imposta pela situação externa, uma tentativa frustrada de lidar com os problemas, mas estresse também pode ser definido como um referente, tanto para descrever uma situação de muita tensão quanto para definir a tensão a tal situação (LIPP E ROCHA, 1994).

Na obra "Trabalho e produtivíssimo" Borsoi (2012) menciona que o estresse, é caracterizado por um estado de tensão, isto é, um dos problemas mais comuns que o ser humano enfrenta, o que ocasiona um desequilíbrio intenso no organismo. $O$ estresse em 
professores tem ocasionado discussões acadêmicas e entre as entidades governamentais, empresariais e sindicais, em razão das austeridades de suas consequências.

Nesse sentido, Carlotto (2012) vem buscando em seus estudos identificar a existência de fatores associados à síndrome de burnout em professores. A autora destaca fatores em dois grupos de variáveis: fatores individuais, que compreendem as variáveis sociodemográficas como sexo, idade, estado civil, situação conjugal, escolaridade, formação, remuneração e fatores de personalidade que abarcam suas características, as estratégias de enfrentamento, a autoeficácia, o lócus de controle, a autoestima, a motivação e a empatia; e os fatores contextuais relacionados ao trabalho em que se consideram carga de trabalho, pressão do tempo, quantidade de alunos e horas trabalhadas, realização de atividades administrativas, relações interpessoais entre gestores, além de aspectos sociais, econômicos e culturais (NOGUEIRA, 2012).

A organização do trabalho do professor na educação básica possui características que o expõem a fatores estressantes que, se persistentes, podem levá-lo a desenvolver a síndrome de burnout (GUGLIELMI E TATROW, 1998). Segundo os autores, a síndrome é um fenômeno complexo, multidimensional, resultante da interação entre os aspectos individuais e o ambiente de trabalho, ou seja, é causada pelos altos níveis de estresse e pelo estado emocional desequilibrado, desenvolvidos a partir de condições de trabalho desgastantes.

A síndrome de burnout quando acometida em professores pode afetar o ambiente educacional e interferir na obtenção dos objetivos pedagógicos, levando estes profissionais a um processo de alienação, desumanização, apatia e ocasionando problemas de saúde e absenteísmo e intenção de abandonar a profissão. De acordo com Batista et al. (2010) a síndrome além de implicar na saúde física e mental do trabalhador, pode impactar também na qualidade do trabalho docente.

A síndrome de burnout é o resultado do estresse crônico, típico do cotidiano do trabalho, principalmente quando neste existem excessiva pressão, conflitos, poucas recompensas emocionais e pouco reconhecimento. Em pesquisas realizadas por Santos e Sobrinho (2012), os resultados identificaram associação entre burnout e características do trabalho docente como o excesso de trabalho, a indisciplina dos alunos, a baixa remuneração, 
a falta de reconhecimento profissional e de reciprocidade na relação com os alunos, o conflito relativo à sobrecarga de papel, a relação professor-família, a presença de dúvidas quanto à competência profissional, problemas de relacionamento com colegas, formação insuficiente para a prática do trabalho e o excesso de responsabilidades.

Segundo Blanca Doménech (1995), no caso do professor da educação básica, o processo é iniciado com uma sensação de inadequação na função e a percepção de ausência de recursos para enfrentar as exigências de seu trabalho. Há sensação de diminuição de sua capacidade de concentração, de resolver problemas e tomar decisões. Como consequência, o professor tende a aumentar o seu esforço, surgindo sinais evidentes de irritação, ansiedade, tensão, medo de não ter sucesso nas aulas, de manter a disciplina, com uma percepção exagerada de suas lacunas e dificuldades.

Estas sensações podem provocar reações de estresse e, se persistentes, podem levar à síndrome de burnout, considerada por Harrison (1999) como um tipo de estresse de caráter persistente vinculado a situações de trabalho, resultante da constante e repetitiva pressão emocional associada com intenso envolvimento com pessoas por longos períodos. E como consequências do burnout, os docentes se afastam do emprego por problemas de saúde, mudam de profissão ou apresentam algum problema de saúde.

Contudo, a síndrome de burnout em profissionais da educação vem recebendo atenção cada vez maior por parte dos pesquisadores (ANDRADE, 2012; SILVA E CARLOTTO, 2003) porém, apesar dos avanços teóricos sobre as relações entre trabalho e saúde dos docentes, no Brasil, a produção científica ainda se apresenta incipiente (GOMES, 2015) e instável.

\section{CONSIDERAÇÕES FINAIS}

Os artigos aqui estudados, mostraram a influência significativa da síndrome de burnout em professores da educação básica, doença ocupacional causada pelo estresse crônico oriundo do trabalho. $\mathrm{O}$ docente integra um grupo de risco em relação à síndrome, $\mathrm{e}$ que necessita de mais investimentos na saúde e qualidade de vida do professor. Melamed et al. (2006) ressalvam que os resultados dos estudos sobre a síndrome de burnout deveriam ser considerados para fomentar políticas de saúde, porque investir na prevenção da saúde e na qualidade de vida do professor é também investir numa educação de qualidade. 
A síndrome de burnout em professores da educação básica pode ser considerada como um fenômeno complexo e multifacetado resultante do entrelaçamento entre diversos aspectos individuais e do ambiente profissional. Estudos revelam que o número elevado de disciplinas ministradas e a existência de qualquer doença de base nos professores são condições associadas significativamente com a síndrome de burnout.

A síndrome de burnout é mais comum em profissionais que atuam constantemente sob pressão tal como, os professores, pois exercem sua profissão com longas jornadas de trabalho, em ambientes de competitividade e com grandes responsabilidades. Autores como Christina Maslach e Mary Sandra Carlotto, descrevem que a síndrome de burnout acomete com mais frequência profissionais que enfrentam dupla jornada, por exemplo, os profissionais da educação da básica.

Mesmo com diversas produções que retratam o perfil e quadro de saúde de professores, pôde-se verificar que, embora tenha ocorrido um discreto aumento de publicações ao longo dos anos, os estudos científicos ainda são escassos, principalmente aqueles relacionados aos docentes que atuam na educação básica. Seria relevante, ainda, a realização de mais pesquisas que abordem o ambiente físico escolar, à cultura, às normas e a relação entre gestores e profissionais, além da interação trabalho-família, com vista a importância dessa variável no contexto laboral atual.

Portanto, a variável estresse ou eventos estressores merece maior atenção por parte dos pesquisadores, que realizam estudos sobre a relação burnout, qualidade de vida e qualidade de vida no trabalho. Buscando identificar demais fatores associados à síndrome de burnout em professores no Brasil. Este estudo, buscou discutir as possíveis causas da síndrome de burnout em docentes da educação básica.

\section{REFERÊNCIAS}

ANDRADE, Lucas Veras de. Mal-estar e atividade docente: um estudo com professoras de $\begin{array}{lllll}\text { educação infantil. Trabalho } \quad \& \quad \text { Educação, } & \text { 2I(I), } & 2012 .\end{array}$ https://periodicos.ufmg.br/index.php/trabedu/article/view/8726/623I

BATISTA, Jaqueline Brito Vital; CARLOTTO, Mary Sandra; COUTINHO, Antônio Souto; AUGUSTO, Lia Giraldo da Silva. Prevalência da síndrome de burnout e fatores sociodemográficos e laborais em professores de escolas municipais da cidade de João Pessoa,
PB. Revista
Brasileira
de
Epidemiologia,
I3(3),
2010. 
https://www.scielo.br/j/rbepid/a/74MV 3 CfF 8g6vSHjWMQJFqkp/?format=pdf\&lang=p $\mathrm{t}$

BORSOI, Izabel Cristina Ferreira . Trabalho e produtivíssimo: saúde e modo de vida de docentes de instituições públicas de Ensino Superior. Caderno de Psicologia Social eTrabalho,I5(I): 2012. http://doi.org/ıo.Ir6o6/issn.198I-0490.vi5iip8I-Ioo

CARLOTTO, Mary Sandra. Síndrome de Burnout em professores: avaliação, fatores associados e intervenção. Porto: LivPsic, 2012. https://doi.org/10.1590/Soro237722011000400003

CARLOTTO, Mary Sandra; CÂMARA, Sheila Gonçalves. Análise da produção científica sobre a síndrome de Burnout no Brasil. Psico, 39(2), 2008. https://revistaseletronicas.pucrs.br/ojs/index.php/revistapsico/article/view/I46I/3035

CODO, Wanderley; VASQUES-MENEZES, Ione. O que é burnout? Em Wanderley Codo (Org.). Educação: Carinho e trabalho (pp. 237-255). Rio de Janeiro: Vozes, 1999.

DOMÉNECH, Blanca Delgado. Introduccion al síndrome "burnout" en profesores y maestros $y$ su abordaje terapeutico. Psicologia Educativa, I(I), I995. https://journals.copmadrid.org/psed/art/ıogd2dd36o8f669car7920c5IIc2a4Ie

GIL-MONTE, Pedro. Magnitude of relationship between burnout and absenteeism: a $\begin{array}{llll}\text { preliminary study. Psychological } & \text { Reports, } & \text { I02(2), }\end{array}$ https://doi.org/10.2466/pro.102.2.465-468

GOMES, Tânia Maria de Oliveira. O relato profissional à luz da metarreflexão: interstícios e confluências no fazer acadêmico-docente. Revista Fundamentos, 2(I), 2015. https://revistas.ufpi.br/index.php/fundamentos/article/view/3764/2183

GUGLIELMI, Sergio.; TATROW, Kristin. Ocupational stress, burnout, and health in teachers: a methodological and theoretical analysis. Review of Education Research, 68(I), 1998. https://doi.org/10.3102/00346543068001061

HARRISON, B. J. Are you to burn out? Fund Raising Management, 30(3), 1999.

LEITER, Michael; MASLACH, Christina. Interventions to prevent and alleviate burnout. In: Michael Leiter, Arnold Bakker e Christina Maslach (Eds.). Burnout at work: a psychological perspective. (pp. 145-167). Hove: Psychology Press, 2014.

LIORENT, Vicente; RUIZ-CALZADO, Inmaculada. Burnout and its relation to sociodemographic variables among education professionals working with people with disabilities in Córdoba (Spain). Ciência Saúde Coletiva, 2I(Io), 2016. http://dx.doi.org/10.1590/1413-812320152110.00732015

LIPP, Marilda; ROCHA, João Carlos. Stress, hipertensão arterial e qualidade de vida: Um guia de tratamento ao hipertenso. Campinas: Papirus, 1994. 
MASLACH, Christina. Job burnout. Annual Review of Psychology, 52(I), 200r. http://doi: Io.II46/annurev.psych.52.1.397

MASLACH, Christina. Understanding Job Burnout. In: Ana Maria Rossi, Pamela Perrewe, Sauter Steven. Stress and quality of working life: Current perspectives in Occupational. Health Psychology. Greenwich, Connecticut: Age Publishing; 2006.

MELAMED, Samuel; SHIROM, Arie; TOKER, Sharon; BERLINER, Shlomo; SHAPIRA, Itzhak. Burnout and risk of cardiovascular disease: evidence, possible causal paths, and promising research directions. Psychological bulletin, 132(3), 2006. http://doi: I0.1037/o0332909.132 .3 .327

NOGUEIRA, Isabel. Maria Gomes de melo. Clima de escola. Dissertação (Mestrado em Docência e Gestão da Educação). Universidade Fernando Pessoa, Porto, 2012. http://pepsic.bvsalud.org/scielo.php?script $=$ sci_nlinks\&ref $=1650695 \&$ pid $=$ Si677II68201700020001300054\&lng=pt

RAJAK, Rupesh; CHANDRA, Bibhas. Exploring Predictors of burnout and work engagement among teachers - A Review on higher educational institutions of India. Journal of the Indian Academy of Applied Psychology, 43(I), 2017. https://psycnet.apa.org/record/2017-10283-017

SANTOS, Alaíde Almeida dos; SOBRINHO, Carlito Lopes Nascimento. Revisão sistemática da prevalência da síndrome de Burnout em professores do ensino fundamental e médio. Revista Baiana de Saúde Pública, 35(2), 2012. https://doi.org/10.22278/23182660.2011.v35.n2.0307

SILVA, Graziela Nascimento da; CARLOTTO, Mary Sandra. Síndrome de Burnout: um estudo com professores da rede pública. Psicologia Escolar e Educacional (ABRAPEE), 7(2), 2003. https://doi.org/10.1590/Si413-85572003000200004 